\title{
EFFECT OF CYLINDRICAL TEXTURE ON DYNAMiC CHARACTERISTICS OF JOURNAL BEARING
}

\author{
T S Reddy Ganji ${ }^{1}$ and S K Kakoty ${ }^{2}$ \\ ${ }^{1}$ Department of Mechanical Engineering, Indian Institute of Technology Guwahati, \\ Assam, India \\ ${ }^{2}$ Department of Mechanical Engineering, Indian Institute of Technology Guwahati, \\ Assam, India
}

\begin{abstract}
Effect of cylindrical texture on dynamic characteristics of hydrodynamic journal bearing is presented in this paper. The Reynolds equation is discretized by finite difference method and solved numerically in an iterative scheme satisfying the appropriate boundary conditions. Stiffness and damping coefficients of fluid film and stability parameters are found using the first-order perturbation method for different eccentricity ratios and various texture parameters like texture depth and texture portion. From the present study, it has found that cylindrical texture exhibits better stability than plain journal bearing.
\end{abstract}

\section{KEYWORDS}

Cylindrical Texture, Stiffness and Damping coefficients, Hydro Dynamic Lubrication, Mass parameter, Journal Bearing

\section{NOMENCLATURE}

$C$ Radial clearance (m)

$D$ Diameter of the bearing (m)

$D_{r r}, D_{\phi \phi}, D_{r \phi}, D_{\phi r}$ Damping coefficients (Ns/m)

$\bar{D}_{r r}, \bar{D}_{\phi \phi}, \bar{D}_{r \phi}, \bar{D}_{\phi r}$ Non-dimensional damping coefficients, $\bar{D}_{i j}=D_{i j} C^{3} / \mu R^{3} L$

$e, \varepsilon$ Eccentricity, $\varepsilon=e / C$

$e_{0}, \varepsilon_{0}$ Steady-state eccentricity, $\varepsilon_{0}=e_{0} / C$

$e_{1}, \varepsilon_{1}$ Perturbed eccentricity, $\varepsilon_{1}=e_{1} / C$

$h, \bar{h}$ Film thickness, $\bar{h}=h / C$

$\Delta h$ Variation of film thickness due to the presence of the texture (m)

$\overline{\Delta h}$ Dimensionless Variation of film thickness due to the presence of the texture, $\overline{\Delta h}=\Delta h / C$

$L$ Bearing length

$M, \bar{M}$ Mass parameter, $\bar{M}=M C \omega^{2} / W_{0}$

DOI : 10.14810/ijmech.2014.3410 
$p, \bar{p}$ Lubricant Pressure, $\bar{p}=p C^{2} / 6 \eta U R$

$\bar{p}_{0}, \bar{p}_{1}, \bar{p}_{2}$ Steady-state and perturbed dimensionless pressures

$R$ Journal radius $(\mathrm{m})$

$r_{p}$ Base radius of dimple

$r_{1}$ Half-length of imaginary textured square cell

$r_{x}, r_{y}, r_{z}$ Texture dimensions along $\mathrm{x}, \mathrm{y}$ and $\mathrm{z}$ directions

$S$ Sommerfeld number, $S=(\eta N / p) \times(R / C)^{2}$

$S_{p}$ Texture area density, $S_{p}=\pi r_{p}^{2} / 4 r_{1}^{2}$

$U$ Linear velocity $(\mathrm{m} / \mathrm{s})$

$W$ Load carrying capacity $(\mathrm{N})$

$\bar{W}$ Dimensionless Load carrying capacity, $\bar{W}=W C^{2} / 6 \eta U R^{2} L$

$x, z$ Cartesian coordinates

$\bar{x}, \bar{z}$ Dimensionless Cartesian coordinates

$x_{1}, z_{1}$ Local coordinates with their origin at the center of a single dimple cell

$\overline{x_{1}}, \overline{z_{1}}$ Local dimensionless coordinates with their origin at the center of a single dimple cell

$\alpha$ Textured portion in circumferential direction

$\beta$ Textured portion in axial direction

$\phi$ Attitude angle

$\eta$ Dynamic viscosity (Pas)

$\mu$ Coefficient of friction

$\bar{\mu}$ Friction variable, $\bar{\mu}=\mu(R / C)$

$\theta$ Angular coordinate

$t$ Time (s)

$\omega, \omega_{p}$ Journal rotational speed ( $\left.\mathrm{rad} / \mathrm{s}\right)$, frequency of journal vibration

$\tau$ Non dimensional time, $\tau=\omega_{p} t$

$\lambda$ Whirl ratio, $\lambda=\omega_{p} / \omega$

$\Lambda$ Bearing number, $\Lambda=6 \eta \omega / p(C / R)^{2}$

()$_{0}$ Steady state value

\section{Subscripts}

$\max$ : Maximum value

min : Minimum value

\section{INTRODUCTION}

Micro dimples are developed by incremental stamping using the structured tool. The structured tool is manufactured by focused ion beam sputtering. Interference lithography is also used for 
producing micro dimples on surfaces. Matsumura et al. [1] have studied and developed micro fabrication techniques on cylinder surface. Matsumura et al. [2] have also developed some micro

dimples on aluminum plates. Tala Ighil et al. [3] presented an analysis of cylindrical textured bearing and showed that the performance

of bearing is influenced by textured surface. The dynamic characteristics of hydrodynamic journal bearings lubricated with micro polar fluids are presented by Das et al. [4]. Brizmer and Kligerman[5] have found that both load capacity and attitude angle of the journal bearings could be improved by using partial LST mode at low eccentricities.Li and Wang [6] have investigated the influence of the radius of the dimples on the tribological performance of a journal bearing. Results showed that the friction coefficient increased with both the width and the height of bulges in the case of journal bearing under light and moderate loading conditions. A linearized perturbation approach has been used by Pai et al. [7] to study the stability characteristics of tritaper journal using the Reynolds boundary condition

The present work aims to find the dynamic characteristics of positive cylindrical textured journal bearing, i.e. the effect of texturing on dynamic characteristics like Mass parameter, Whirl ratio, Stiffness and Damping coefficients.

\section{NUMERICAL FORMULATION}

The Reynolds equation in non-dimensional form for an incompressible fluid can be written as

$$
\frac{\partial}{\partial \theta}\left(\bar{h}^{3} \frac{\partial \bar{p}}{\partial \theta}\right)+\left(\frac{D}{L}\right)^{2}\left(\bar{h}^{3} \frac{\partial^{2} \bar{p}}{\partial \bar{z}^{2}}\right)=\frac{\partial \bar{h}}{\partial \theta}+2 \lambda \frac{\partial \bar{h}}{\partial \tau}
$$

Where,

$$
\theta=\frac{x}{R}, \quad \bar{z}=\frac{z}{L / 2}, \quad \bar{h}=\frac{h}{C}, \quad \bar{p}=\frac{p C^{2}}{6 \eta U R}, \quad \tau=\omega_{p} t, \quad \lambda=\frac{\omega_{p}}{\omega}
$$

The film thickness for textured journal bearing, $h$, can be written as follows:

$h_{0}=h_{\text {smooth }}(\theta)-\Delta h(\theta, z)$ 


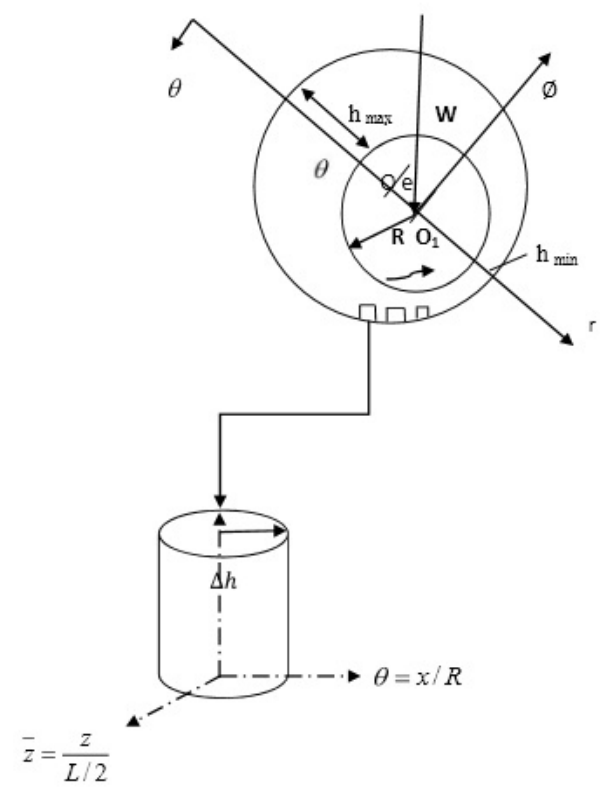

Figure 1: Cylindrical Textured Journal Bearing

Non dimensional film thickness can be written as

$$
\begin{array}{ll}
\overline{h_{0}}=1+\varepsilon_{0} \cos \theta-\overline{\Delta h} & \text { if }{\overline{x_{1}}}^{2}+\bar{z}_{1}^{2} \leq 1 \\
\overline{h_{0}}=1+\varepsilon_{0} \cos \theta & \text { if }{\overline{x_{1}}}^{2}+{\overline{z_{1}}}^{2}>1
\end{array}
$$

The pressure and film thickness can be expressed for small amplitude of vibration as $\bar{p}=\bar{p}_{0}+\varepsilon_{1} e^{i \tau} \bar{p}_{1}+\varepsilon_{0} \phi_{1} e^{i \tau} \bar{p}_{2}$,

$\bar{h}=\bar{h}_{0}+\varepsilon_{1} e^{i \tau} \cos \theta+\varepsilon_{0} \phi_{1} e^{i \tau} \sin \theta$

The following three equations are obtained by substituting equation (5) into equation (1) and retaining the first order terms and equating the coefficients of $\varepsilon_{0}, \varepsilon_{1} e^{i \tau}$ and $\varepsilon_{0} \phi_{1} e^{i \tau}$

$\frac{\partial}{\partial \theta}\left(\bar{h}_{0}^{3} \frac{\partial \overline{p_{0}}}{\partial \theta}\right)+\left(\frac{D}{L}\right)^{2} \frac{\partial}{\partial \bar{z}}\left(\bar{h}_{0}^{3} \frac{\partial \overline{p_{0}}}{\partial \bar{z}}\right)=\Lambda \frac{\partial \overline{h_{0}}}{\partial \theta}$ 


$$
\begin{aligned}
& \frac{\partial}{\partial \theta}\left(\bar{h}_{0}^{3} \frac{\partial \overline{p_{1}}}{\partial \theta}\right)+\left(\frac{D}{L}\right)^{2} \frac{\partial}{\partial \bar{z}}\left(\bar{h}_{0}^{3} \frac{\partial \overline{p_{1}}}{\partial \bar{z}}\right)+3 \frac{\partial}{\partial \theta}\left(\bar{h}_{0}^{2} \frac{\partial \overline{p_{0}}}{\partial \theta} \cos \theta\right) \\
& +\left(\frac{D}{L}\right)^{2} \frac{\partial}{\partial \bar{z}}\left(\bar{h}_{0}^{2} \frac{\partial \overline{p_{0}}}{\partial \bar{z}} \cos \theta\right)=-\Lambda \sin \theta+i 2 \Lambda \lambda \cos \theta \\
& \frac{\partial}{\partial \theta}\left(\bar{h}_{0}^{3} \frac{\partial \overline{p_{2}}}{\partial \theta}\right)+\left(\frac{D}{L}\right)^{2} \frac{\partial}{\partial \bar{z}}\left(\bar{h}_{0}^{3} \frac{\partial \overline{p_{2}}}{\partial \bar{z}}\right)+3 \frac{\partial}{\partial \theta}\left(\bar{h}_{0}^{2} \frac{\partial \overline{p_{0}}}{\partial \theta} \sin \theta\right) \\
& +\left(\frac{D}{L}\right)^{2} \frac{\partial}{\partial \bar{z}}\left(\bar{h}_{0}^{2} \frac{\partial \overline{p_{0}}}{\partial \bar{z}} \sin \theta\right)=\Lambda \cos \theta+i 2 \Lambda \lambda \sin \theta
\end{aligned}
$$

Boundary conditions used for steady state and dynamic pressures are

$$
\overline{p_{i}}(\theta, 0)=\bar{p}_{i}(\theta, L)=0 \bar{p}_{i}(\theta, \bar{z})=\overline{p_{i}}(2 \pi, \bar{z}) \quad \text { Where } \overline{p_{i}}=\overline{p_{0}}, \overline{p_{1}}, \overline{p_{2}}
$$

The boundary conditions shown above should be complemented by the conditions at the boundaries of possible cavitation regions associated with each individual dimple. The Reynolds boundary condition (also known as the Swift-Stieber boundary condition) implies that the pressure gradient with respect to the direction normal to the boundary of the cavitation zone is zero and the dimensionless pressure inside the cavitation zone is also zero. Using an iterative solution scheme, it is simple to apply this condition to the Equations (6,7 and 8). Negative, i.e., sub-ambient pressures are changed to zero in each iterative cycle; the process converges, by numerical diffusion, to the required Reynolds condition [8].

Figure 1 shows the schematic representation of textured journal bearing. The texture shape used in this analysis is cylindrical. The texture distribution is uniform as shown in Fig. 2. When $r_{p}$ represents the radius of cylindrical dimple, $2 \mathrm{r}_{1}$ is the length of the imaginary square cell as shown in the Fig. 2. Equations (6, 7 and 8) are solved numerically in a finite difference grid. GaussSiedel method with over relaxation has been used for solving the discretized Reynolds equations satisfying the boundary conditions.

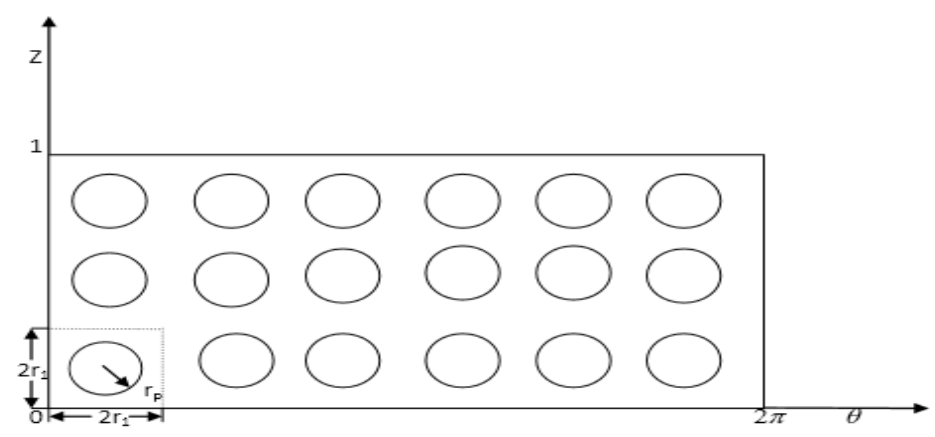

Figure 2: Distribution of textures on the bearing surface 
As the dimples are very small, it has been observed that finite difference grid needs to be very fine for convergence. A grid size of $350 \times 50$ in the present work has been found to give gridindependent pressure distribution. The components of steady-state load carrying capacity are estimated by numerical integration of pressure as shown in equations ( 9 and 10). Steady state load and attitude angles are estimated as shown in equation (11 and 12).

$$
\begin{aligned}
& \bar{W}_{r}=-\int_{0}^{1} \int_{0}^{2 \pi} \bar{p}_{0} \cos \theta d \theta d \bar{z} \\
& \bar{W}_{t}=\int_{0}^{1} \int_{0}^{2 \pi} \bar{p}_{0} \sin \theta d \theta d \bar{z} \\
& \bar{W}_{0}=\sqrt{\bar{W}_{r}^{2}+\bar{W}_{t}^{2}} \\
& \phi_{0}=\tan ^{-1}\left(\frac{\bar{W}_{t}}{\bar{W}_{r}}\right)
\end{aligned}
$$

The expression for friction variable can be written as [9]

$$
\bar{\mu}=\mu\left(\frac{R}{C}\right)=\frac{\int_{0}^{2 \pi}(3 \bar{h}(\partial \bar{p} / \partial \theta)+1 / \bar{h}) d \theta}{6 \bar{W}}
$$

The stiffness and damping coefficients are given by

$$
\begin{aligned}
& \bar{K}_{r r}=-\operatorname{Re}\left(\int_{0}^{1} \int_{0}^{2 \pi} \bar{p}_{1} \cos \theta d \theta d \bar{z}\right) \\
& \bar{K}_{\phi r}=-\operatorname{Re}\left(\int_{0}^{1} \int_{0}^{2 \pi} \bar{p}_{1} \sin \theta d \theta d \bar{z}\right) \\
& \bar{D}_{r r}=-\operatorname{Im} \frac{\left(\int_{0}^{1} \int_{0}^{2 \pi} \bar{p}_{1} \cos \theta d \theta d \bar{z}\right)}{\lambda} \\
& \bar{D}_{\phi r}=-\operatorname{Im} \frac{\left(\int_{0}^{1} \int_{0}^{2 \pi} \bar{p}_{1} \sin \theta d \theta d \bar{z}\right)}{\lambda} \\
& \bar{K}_{\phi \phi}=-\operatorname{Re}\left(\int_{0}^{1} \int_{0}^{2 \pi} \bar{p}_{2} \sin \theta d \theta d \bar{z}\right) \\
& \bar{K}_{r \phi}=-\operatorname{Re}\left(\int_{0}^{1} \int_{0}^{2 \pi} \bar{p}_{2} \cos \theta d \theta d \bar{z}\right) \\
& \bar{D}_{\phi \phi}=-\operatorname{Im} \frac{\left(\int_{0}^{1} \int_{0}^{2 \pi} \bar{p}_{2} \sin \theta d \theta d \bar{z}\right)}{\lambda} \\
& \bar{D}_{r \phi}=-\operatorname{Im} \frac{\left(\int_{0}^{1} \int_{0}^{2 \pi} \bar{p}_{2} \cos \theta d \theta d \bar{z}\right)}{\lambda}
\end{aligned}
$$


Stiffness and damping coefficients are used in the equations of motion. The equations of motion in non-dimensional form can be written as [10]:

$$
\begin{aligned}
& \bar{M}=\frac{1}{\lambda^{2}\left(\bar{D}_{\phi \phi}+\bar{D}_{r r}\right)}\left[\begin{array}{c}
\left(\bar{K}_{r r} \bar{D}_{\phi \phi}+\bar{D}_{r r} \bar{K}_{\phi \phi}\right)-\left(\bar{K}_{\phi r} \bar{D}_{r \phi}+\bar{D}_{\phi r} \bar{K}_{r \phi}\right) \\
+\frac{\bar{W}}{\mathcal{E}_{0}}\left(\bar{D}_{r r} \cos \phi_{0}-\bar{D}_{\phi r} \sin \phi_{0}\right)
\end{array}\right] \\
& \bar{M}^{2} \lambda^{4}-\lambda^{2}\left[\bar{M}\left(\frac{\bar{W} \cos \phi_{0}}{\varepsilon_{0}}+\bar{K}_{\phi \phi}+\bar{K}_{r r}\right)+\left(\bar{D}_{r r} \bar{D}_{\phi \phi}-\bar{D}_{\phi r} \bar{D}_{r \phi}\right)\right] \\
& +\left(\bar{K}_{r r} \bar{K}_{\phi \phi}-\bar{K}_{\phi r} \bar{K}_{r \phi}\right)+\frac{\bar{W}}{\varepsilon_{0}}\left(\bar{K}_{r r} \cos \phi_{0}-\bar{K}_{\phi r} \sin \phi_{0}\right)=0
\end{aligned}
$$

Equations (15) and (16) are linear algebraic equations in $\bar{M}$ and $\lambda$. Solution ofthesewillgive $\bar{M}$ and $\lambda$.

\section{RESULTS AND DISCUSSIONS}

\subsection{Characteristics of journal bearing}

The dynamic characteristics are presented in this section. Direct and cross stiffness coefficients for increasing texture depth are presented in Fig. 3, when direct and cross damping coefficients are presented in Fig. 4. The stability margin and whirl ratio have been presented in Fig. 5. These results are for $L / D=1, \varepsilon=0.3, S_{p}=0.8, \alpha=1, \beta=1$. It has been observed from Fig. 3 that the non-dimensional direct stiffness coefficient, $\bar{K}_{\phi \phi}$ increases with increase in texture depth and $\bar{K}_{r r}, \bar{K}_{r \phi}$ and decreases slightly with increase in texture depth. The non-dimensional cross stiffness coefficient, $\bar{K}_{\phi r}$ increases slightly with increase in texture depth. Further it has been observed from Fig. 4 that the non-dimensional direct damping coefficient, $\bar{D}_{\phi \phi}$ increases with increase in texture depth and $\bar{D}_{r r}$, decreases slightly with increase in texture depth. The nondimensional cross damping coefficients $\bar{D}_{\phi r}$ and $\bar{D}_{r \phi}$ decrease slightly with increase in texture depth. 
International Journal of Recent advances in Mechanical Engineering (IJMECH) Vol.3, No.4, November 2014

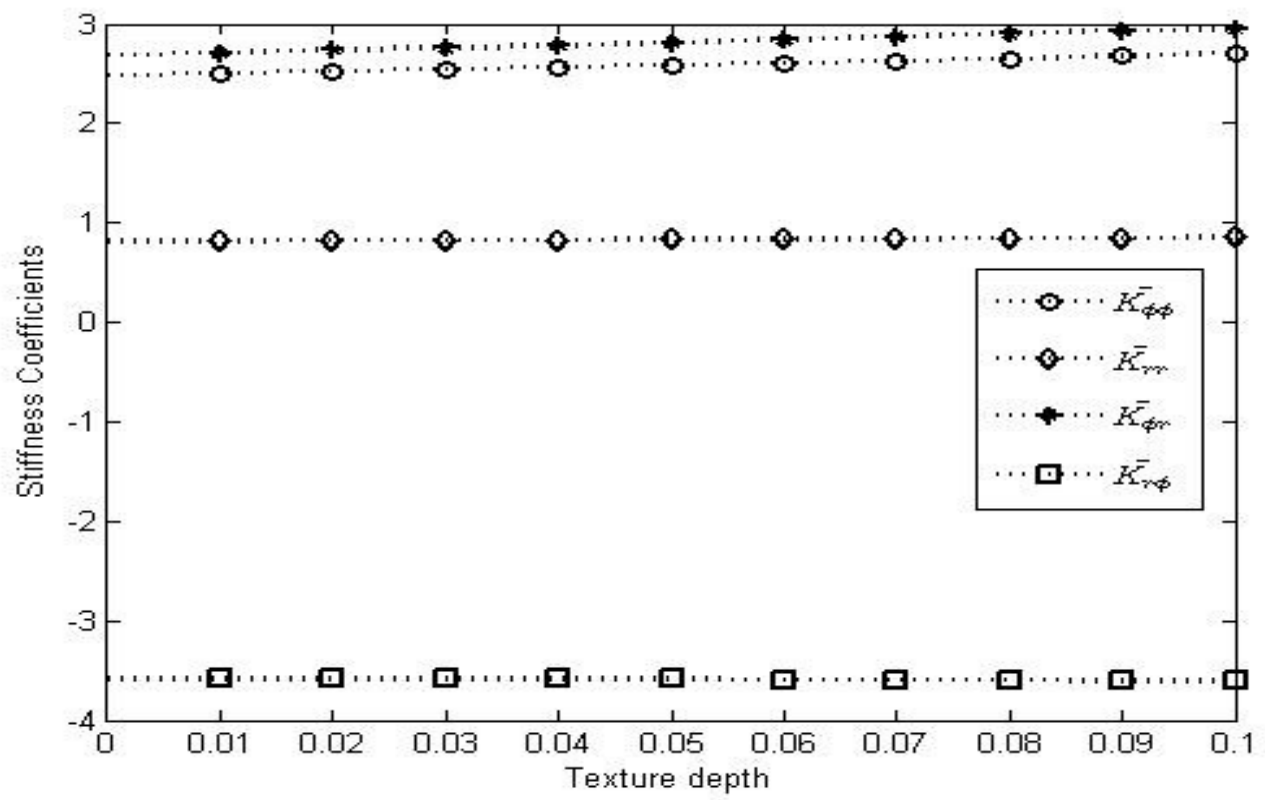

Figure 3: Stiffness coefficients vs. Texture depth $\left(L / D=1, \varepsilon=0.3, S_{p}=0.7, \alpha=1, \beta=1\right)$

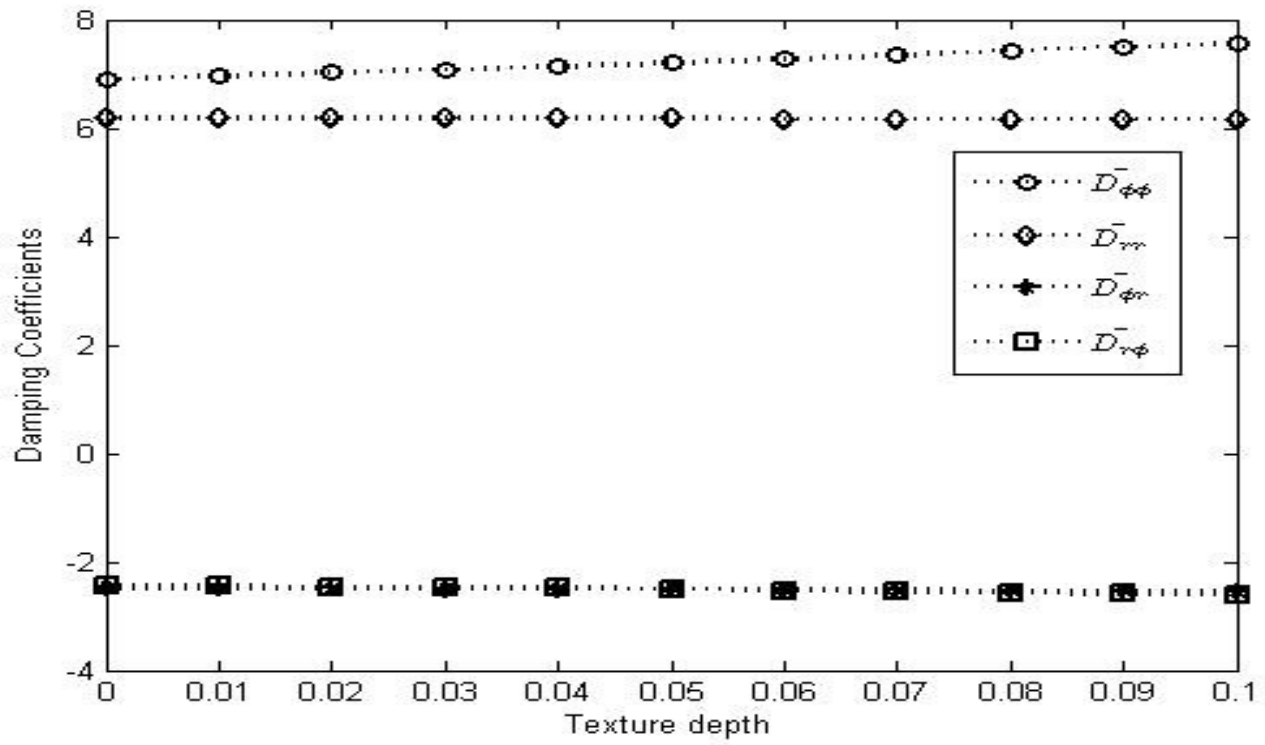

Figure 4: Damping coefficients vs. Texture depth $\left(L / D=1, \mathcal{E}=0.3, S_{p}=0.7, \alpha=1, \beta=1\right)$ 


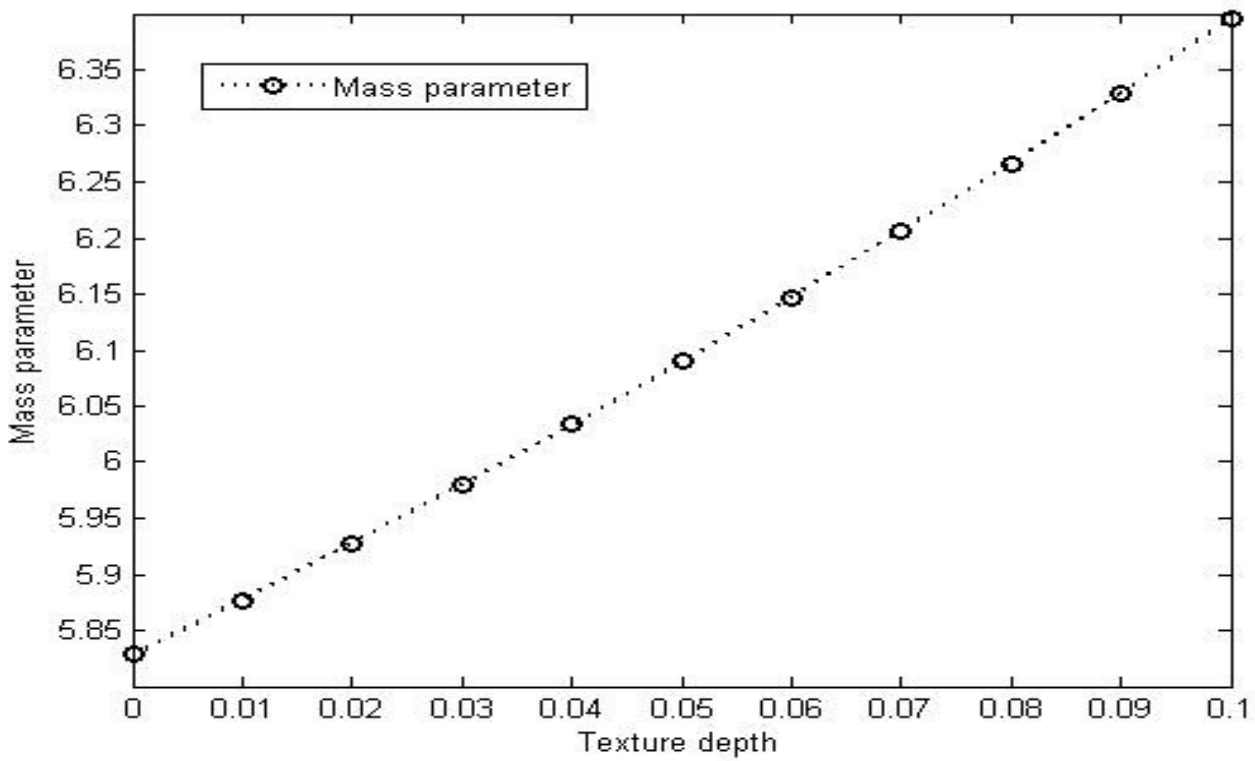

Figure 5: Mass parameter and whirl ratio vs. Texture $\operatorname{depth}\left(L / D=1, \mathcal{E}=0.3, S_{p}=0.7, \alpha=1, \beta=1\right)$

Critical mass parameter, a function of speed, which is the measure of stability threshold, increases with increase in texture depth as seen from Fig. 5. The mass parameter increases with increase in textured depth and it is minimum when texture depth is zero. Texture depth zero indicates plain journal bearing. Therefore, it is inferred that the textured bearing has a better stability characteristics compared to plain journal bearing.

Figure 6represents the variation of non-dimensional stiffness coefficients with eccentricity ratio for $L / D=1, S_{p}=0.7, \Delta \bar{h}=0.1, \alpha=1, \beta=1$. The non-dimensional direct stiffness coefficients, $\bar{K}_{\phi \phi}$ and $\bar{K}_{r r}$ increase slightly with increase in eccentricity ratio. The non-dimensional cross stiffness coefficient, $\bar{K}_{\phi r}$ decreases with increase in eccentricity ratio and $\bar{K}_{r \phi}$ increases with increase in eccentricity ratio. 
International Journal of Recent advances in Mechanical Engineering (IJMECH) Vol.3, No.4, November 2014

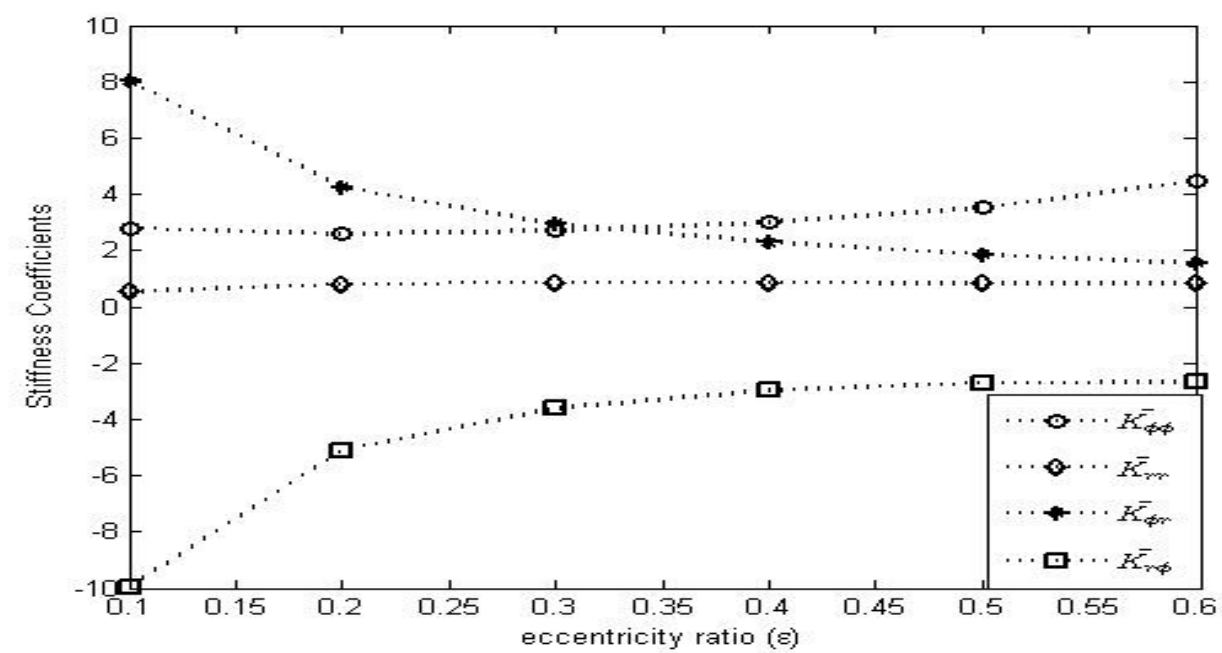

Figure6: Stiffness coefficients vs. Eccentricity $\operatorname{ratio}\left(L / D=1, S_{p}=0.7, \Delta \bar{h}=0.1, \alpha=1, \beta=1\right)$

Figure 7 represents the variation of non-dimensional damping coefficients with eccentricity ratio for $L / D=1, S_{p}=0.7, \Delta \bar{h}=0.1, \alpha=1, \beta=1$. The non-dimensional direct damping coefficients, $\bar{D}_{\phi \phi}$ and $\bar{D}_{r r}$ decrease with increase in eccentricity ratio. The non-dimensional cross damping coefficients, $\bar{D}_{\phi r}$ and $\bar{D}_{r \phi}$ increase slightly with increase in eccentricity ratio. It hasbeen seen from Fig. 8 that the mass parameter increases with increase in eccentricity ratio. Whirl ratio decreases slightly with increase in eccentricity ratio. Textured journal bearing exhibits better stability at higher eccentricity ratios.

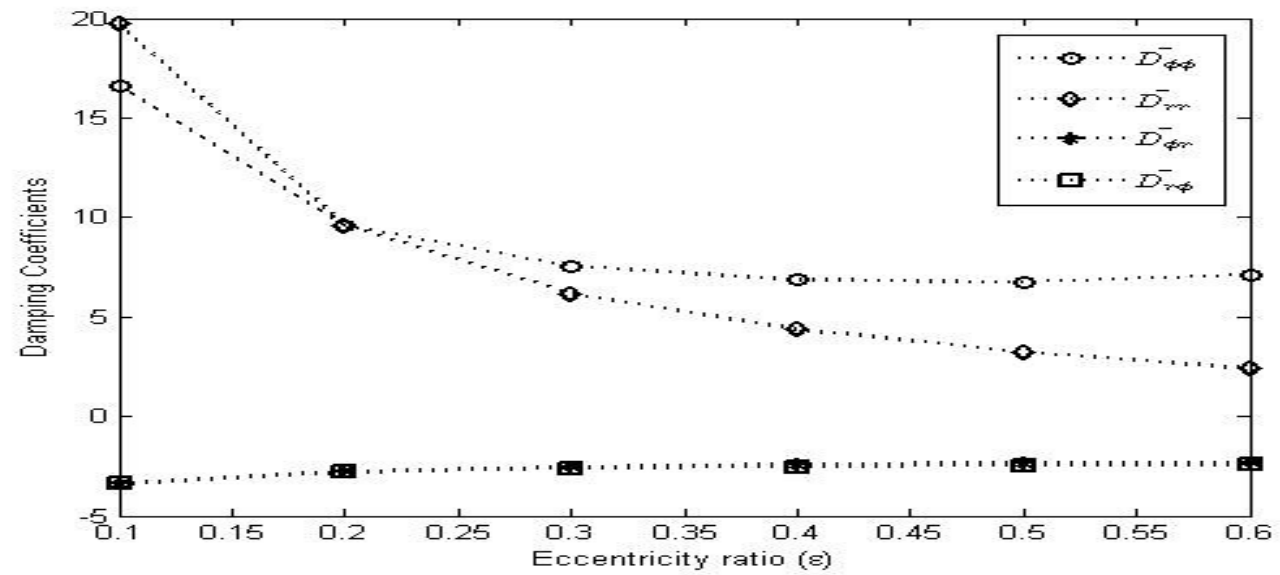

Figure 7:Damping coefficients vs. Eccentricity $\operatorname{ratio}\left(L / D=1, S_{p}=0.7, \Delta \bar{h}=0.1, \alpha=1, \beta=1\right)$ 
International Journal of Recent advances in Mechanical Engineering (IJMECH) Vol.3, No.4, November 2014

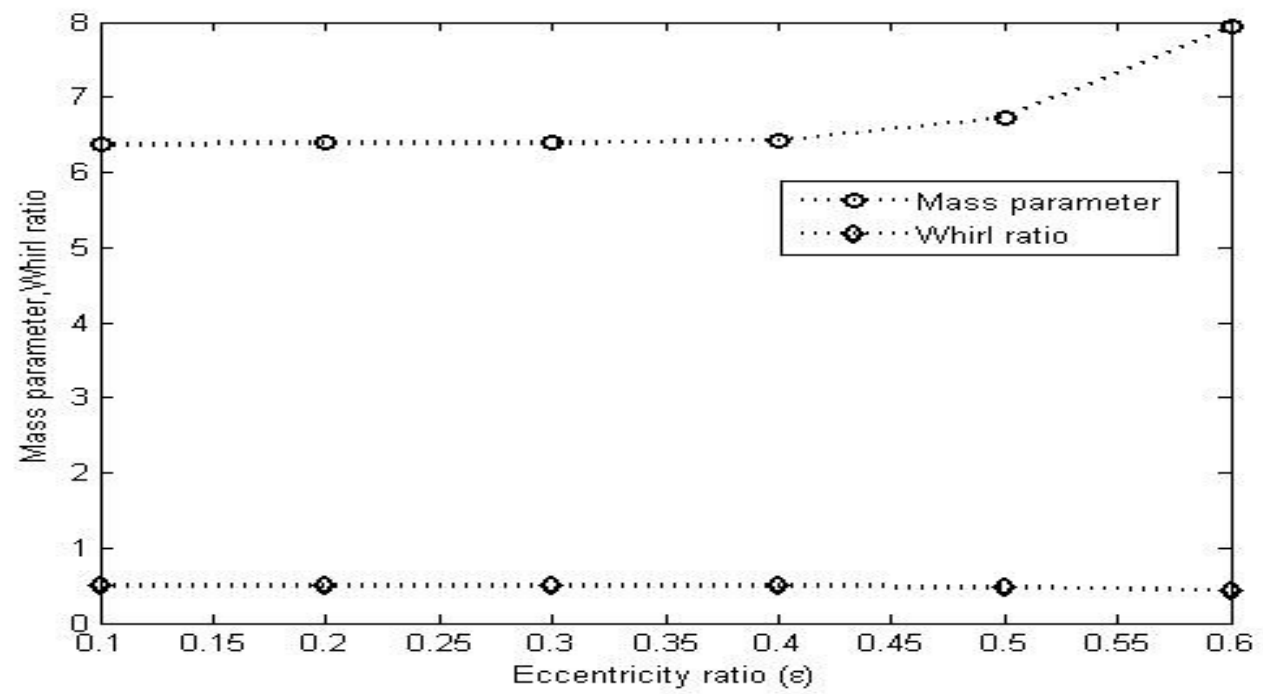

Figure 8: Mass parameter and whirl ratio vs. Eccentricity ratio

$$
\left(L / D=1, S_{p}=0.7, \Delta \bar{h}=0.1, \alpha=1, \beta=1\right)
$$

The effect of texture portion on dynamic coefficients and stability has been presented in Figs. 9, 10 and 11 for $L / D=1, S_{p}=0.7, \Delta \bar{h}=0.1, \varepsilon=0.6, \beta=1$. The maximum values of stiffness and damping coefficients are observed that at $50 \%$ texture portion.

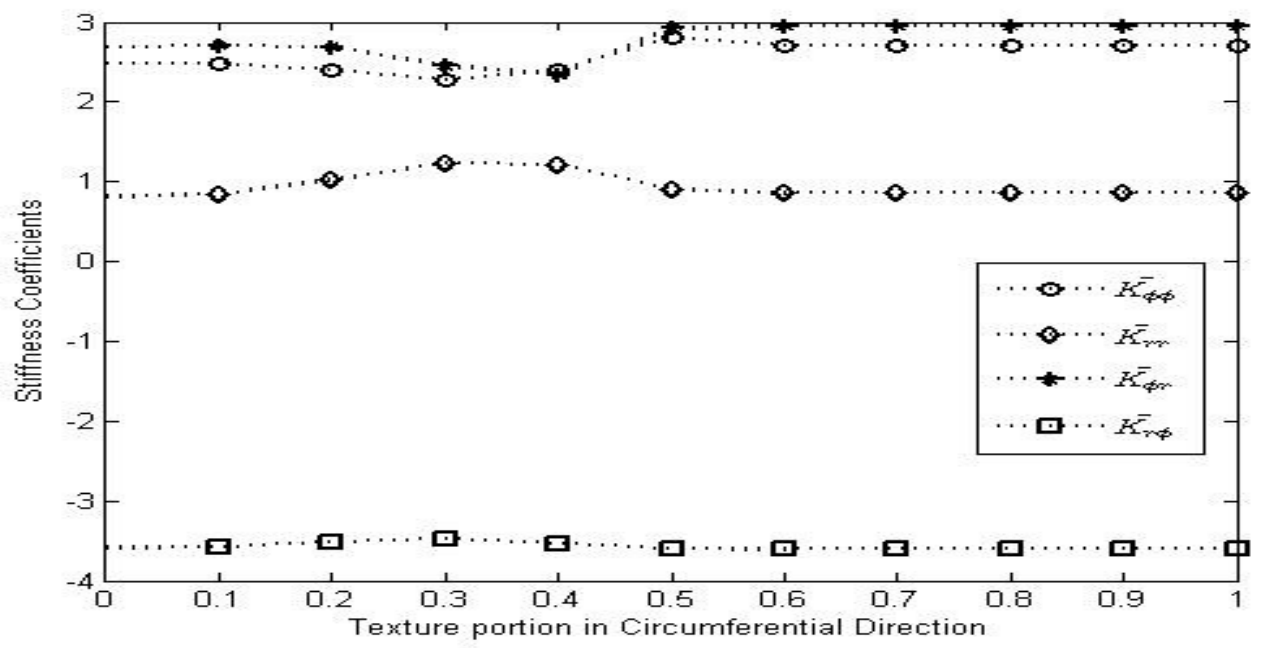

Figure 9: Effect of Textured portion in Circumferential Direction on Stiffness coefficients of Textured Journal Bearing $\left(L / D=1, S_{p}=0.7, \Delta \bar{h}=0.1, \varepsilon=0.6, \beta=1\right)$ 
International Journal of Recent advances in Mechanical Engineering (IJMECH) Vol.3, No.4, November 2014

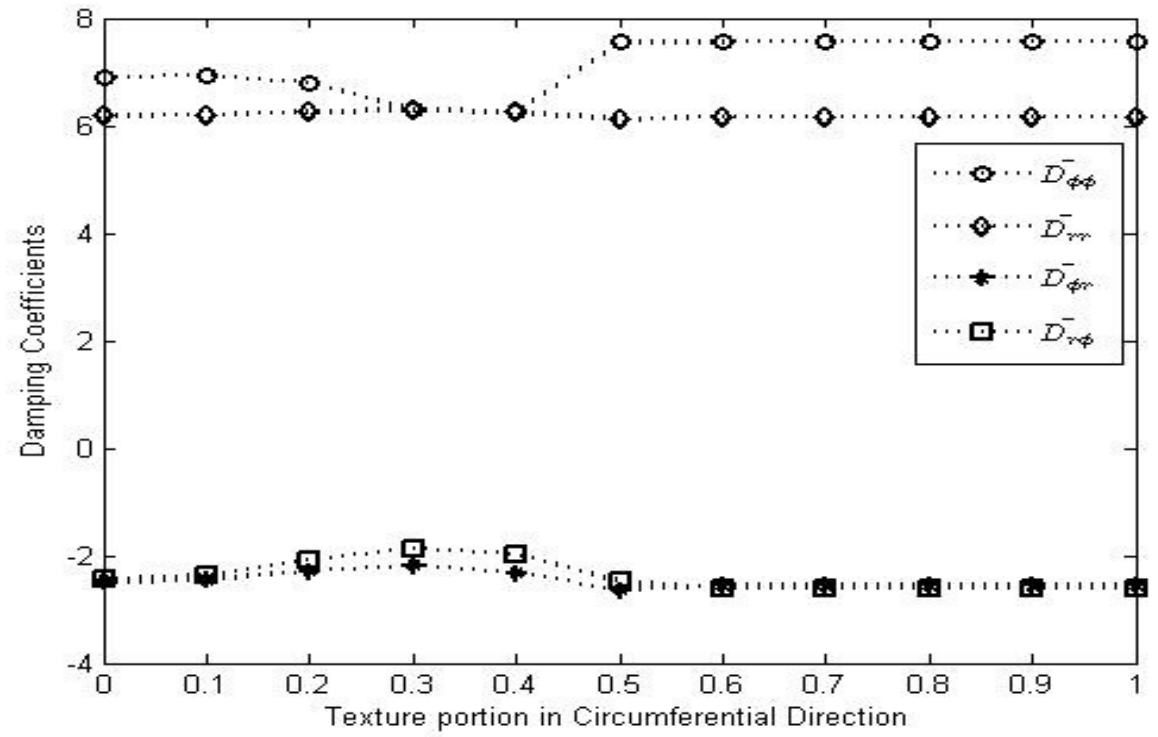

Figure 10: Effect of Textured portion in Circumferential Direction on damping coefficients of Textured Journal Bearing $\left(L / D=1, S_{p}=0.7, \Delta \bar{h}=0.1, \varepsilon=0.6, \beta=1\right)$

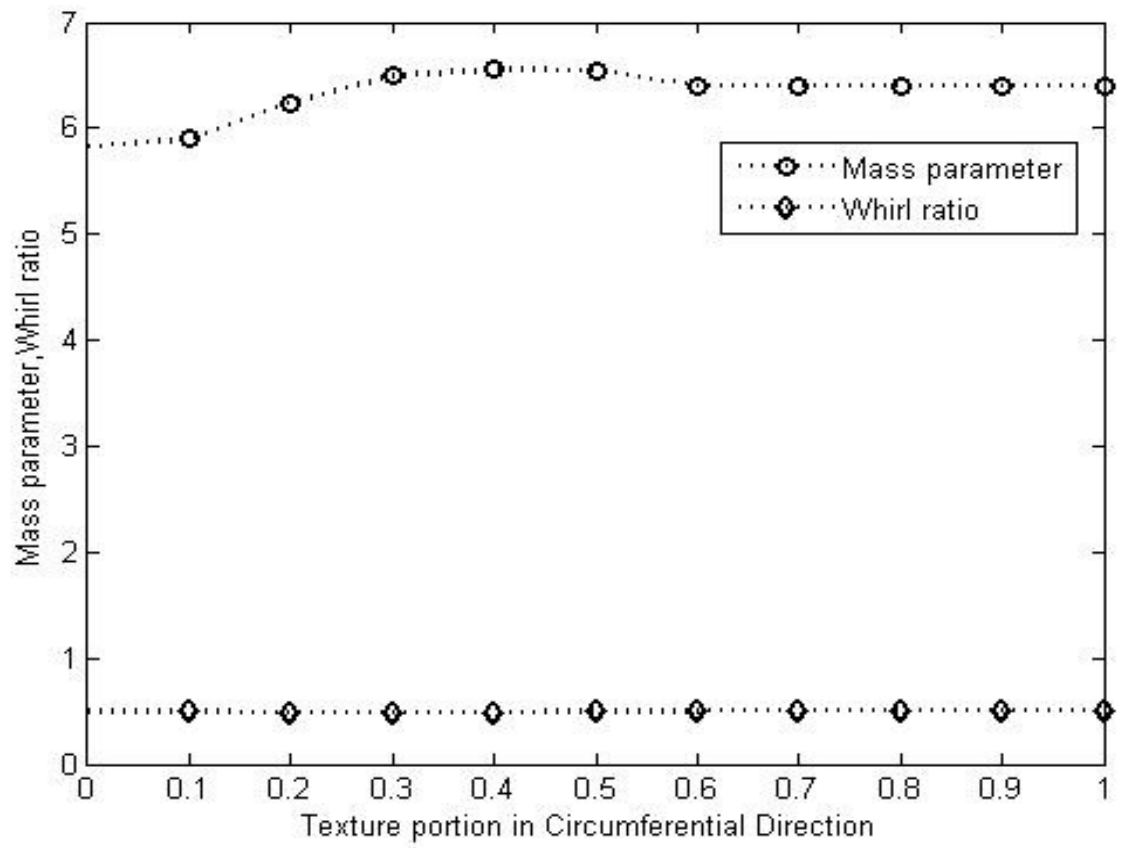

Fig. 11 Effect of Textured portion in Circumferential Direction on Mass parameter and whirl ratio of Textured Journal Bearing $\left(L / D=1, S_{p}=0.7, \Delta \bar{h}=0.1, \varepsilon=0.6, \beta=1\right)$ 


\section{Conclusions}

The dynamic coefficients of cylindrical textured journal bearing are presented for increasing texture depth, eccentricity ratio and texture portion in the foregoing section. Effect of these parameters on stability has also been presented here. Few important observations are

- Increase in texture depth improves the stability of hydrodynamic journal bearing.

- The effect of texture portion on stability is very prominent and the maximum stability is obtained at $50 \%$ texture portion for the operating conditions presented here. However, optimum texture portion at different operating conditions for the maximum stability may be of interest for the bearing designers in future.

\section{REFERENCES}

[1] T. Matsumura, F. Iida, H. Hirosea \& M. Yoshino, (2012) "Micro machining for control of wettability with surface topography", Journal of Materials Processing Technology, Vol. 212, No. 12, pp26692677.

[2] T. Matsumura, H. Sadakata, H. Makihata \& M. Yoshino, (2013) "Micro fabrication on cylinder surface for control of wettability", Journal of Manufacturing Processes, Vol. 15, No. 1, pp8-13.

[3] N. Tala-Ighil, M. Fillon \& P. Maspeyrot, (2011) "Effect of Textured Area on the Performances of a Hydrodynamic Journal Bearing",Tribology International, Vol. 44, No. 3, pp211-219.

[4] S. Das, S.K. Guha \& A.K. Chattopadhyay (2004)“Linear stability analysis of hydrodynamic journal bearingsunder micro polar lubrication”, Tribology International,Vol. 38, No. 5, pp500-507.

[5] V. Brizmer \&Y. Kligerman, (2012) “A Laser Surface Textured Journal Bearing”, Journal of Tribology, Vol. 134, No. 3, pp031702-031709.

[6] J. Li\& X. Wang, (2013) "Numerical Simulation of the Influence of the Bulges around Laser Surface Textures on the Tribological Performance",Tribology Transactions, Vol. 56, No. 6, pp1011-1018.

[7] R. Pai, D. SrikanthRao, B. S. Shenoy\& R. S. Pai, (2012) "Stability Characteristics of a Tri-taper Journal Bearing: a Linearized Perturbation Approach", Journal of Materials Research and Technology,Vol. 1, No. 2, pp84-90.

[8] V. Brizmer, Y. Kligerman \& I. Etsion, (2003) "A laser surface textured parallel thrust bearing", Tribology Transactions, Vol. 46, No. 3, pp397-403.

[9] L. Roy \& S. K. Kakoty, (2013) "Optimum Groove Location of Hydrodynamic Journal Bearing Using Genetic Algorithm”, Advances in Tribology, Vol. 2013, Article ID 580367, pp1-13.

[10] M. VijayaKini, R. S. Pai, D. SrikanthRao, B. S. Shenoy\& R. Pai, (2009) "Effect of Groove Location on the Dynamic Characteristics of Multiple Axial Groove Water Lubricated Journal Bearing", World Academy of Science, Engineering and Technology,Vol. 3, No. 12, pp12-24. 